\title{
Household food insecurity and symptoms of neurologic disorder in Ethiopia: An observational analysis
}

\author{
Abdulrahman M El-Sayed ${ }^{1,2,3^{*}}$, Craig Hadley ${ }^{4}$, Fasil Tessema ${ }^{5}$, Ayelew Tegegn ${ }^{5}$, John A Cowan Jr ${ }^{6}$, Sandro Galea ${ }^{1}$
}

\begin{abstract}
Background: Food insecurity (FI) has been shown to be associated with poor health both in developing and developed countries. Little is known about the relation between FI and neurological disorder. We assessed the relation between $\mathrm{Fl}$ and risk for neurologic symptoms in southwest Ethiopia.

Methods: Data about food security, gender, age, household assets, and self-reported neurologic symptoms were collected from a representative, community-based sample of adults $(N=900)$ in Jimma Zone, Ethiopia. We calculated univariate statistics and used bivariate chi-square tests and multivariate logistic regression models to assess the relation between $\mathrm{Fl}$ and risk of neurologic symptoms including seizures, extremity weakness, extremity numbness, tremors/ataxia, aphasia, carpal tunnel syndrome, vision dysfunction, and spinal pain.
\end{abstract}

Results: In separate multivariate models by outcome and gender, adjusting for age and household socioeconomic status, severe Fl was associated with higher odds of seizures, movement abnormalities, carpal tunnel, vision dysfunction, spinal pain, and comorbid disorders among women. Severe FI was associated with higher odds of seizures, extremity numbness, movement abnormalities, difficulty speaking, carpal tunnel, vision dysfunction, and comorbid disorders among men.

Conclusion: We found that FI was associated with symptoms of neurologic disorder. Given the cross-sectional nature of our study, the directionality of these associations is unclear. Future research should assess causal mechanisms relating $\mathrm{Fl}$ to neurologic symptoms in sub-Saharan Africa.

\section{Background}

Household food insecurity (FI) has been defined as limited or uncertain availability of nutritionally adequate and safe foods or limited or uncertain ability to acquire acceptable foods in socially acceptable ways $[1,2]$. In wealthy countries, it has been estimated that $11.1 \%$ of households are food insecure, with FI higher than 35\% among the poor [1]. FI and related indicators, food insufficiency and hunger, have been associated with decreased adult caloric intake [3-5], psychosocial dysfunction in children [6], overweight in young children [7], increased body weight in women [8,9], sociofamilial dysfunction [10], and lower self-reported physical and mental health [11-14].

\footnotetext{
* Correspondence: ame2145@columbia.edu

'Department of Epidemiology, Columbia University, New York, NY, USA

Full list of author information is available at the end of the article
}

FI, food insufficiency, and hunger are generally thought to be substantially more prevalent in poorer countries than they are in richer countries. Although the literature about the consequences of FI is sparse in less wealthy countries, the extant evidence suggests that the consequences of FI extend well beyond undernutrition $[15,16]$. Ethnographic and epidemiologic studies from sub-Saharan Africa (SSA) suggest that FI is associated with poor mental health $[15,16]$. Hadley and Patil showed that household FI was associated with symptoms of anxiety and depression in rural Tanzania [15], and that seasonal increases in FI were associated with increases in symptoms of depression and anxiety [16]. Hadley and colleagues showed that FI was associated with higher symptoms of depression, anxiety, and post-traumatic stress [17].

One of the potential correlates of FI is neurologic dysfunction (ND). It is estimated that 1 in 9 people worldwide die of a ND, and that ND accounts for $28 \%$ of

\section{C) Biomed Central}


years lived with disability [18]. Several studies have considered the prevalence of ND in Ethiopia. Tamrat and colleagues [19] found that the prevalence of all-cause disability in three rural areas in northern Ethiopia was $4.9 \%$. In particular, the prevalence of epileptic seizure was $0.7 \%$, of vision dysfunction was $1.5 \%$ and of walking difficulty was $1.7 \%$. In a similar study, Fitaw and Boersma [20] assessed the prevalence of disability in one urban and three rural areas in northwestern Ethiopia. They found that the prevalence of disability was $3.8 \%$; $47.0 \%$ of those had lower locomotive disability, $28.6 \%$ had vision dysfunction, $16.1 \%$ had upper motor disability, $10.3 \%$ had cognitive impairments, and $8.3 \%$ had hearing loss, respectively. Tekle-Haimanot and colleagues [21] assessed the incidence of specific neurologic diagnoses in a rural population in central Ethiopia. They found high incidence of the following disorders: epilepsy $(520 / 100,000)$, postpoliomyelitis paralysis $(240 / 100,000)$, mental retardation $(170 / 100,000)$, peripheral neuropathy $(150 / 100,000)$ and deaf-mutism $(130 / 100,000)$. The incidence of hemiparesis was $62 / 100,000$, that of cerebral palsy was $20 / 100,000,16 / 100,000$ for optic atrophy, $12 / 100,000$ for perceptive deafness, 10/100,000 for tropical spastic paraparesis, 7/100,000 for Parkinson's disease and 5/100,000 each for motor neuron disease, ataxia, and chorea/athetosis.

Although there is a paucity of research about specific social and demographic determinants of ND, both behavioral, as well as physiological studies suggest that malnutrition in early neurologic development is a risk factor for ND in later life [22,23]. Considering the determinants of ND in less wealthy countries, the World Health Organization (WHO) suggested that malnutrition, perinatal complications, and advanced parasitic infection are among the most important worldwide determinants of ND. Because structural causes underlying some of the leading risk factors for disabling disorders of the nervous system, such as malnutrition, poor sanitation, and lack of healthcare access are most prevalent in less wealthy countries, populations in these countries are especially at risk $[18,24]$.

In light of the extant research, it is plausible that FI could be a determinant of ND. Alternatively, it is also plausible that those with ND are more likely to be disabled and therefore, less capable of providing themselves and their families with a consistent food supply. This is especially true in rural SSA where subsistence agriculture is the primary means of food accrual. In this analysis, we use data from the Gilgel Gibe Growth and Development Study (GGGDS), a population-representative sample in southwest Ethiopia to assess the relation between FI and symptoms of ND, including spinal pain, extremity weakness, extremity numbness, seizure, vision dysfunction, aphasia, tremors/ataxia, carpal tunnel syndrome, and spinal pain.

\section{Methods \\ Sample}

This study took place in Jimma zone, southwest Ethiopia in the Gilgel Gibe area outside of Jimma Town. This is a predominantly rural area where the primary occupation is subsistence agriculture and FI is chronic.

The Gilgel Gibe Growth and Development Study (GGGDS) is a cohort study of families in the Gilgel Gibe Field Research Center that is concerned with adult mental health, neurologic health, and child development. The study involves questionnaire and anthropometric information collected from the parents, and developmental assessments conducted on their children. We report here on baseline information collected from the parents.

The baseline cohort for the GGGDS was a random sample of households that had a child between the ages of 3-24 months from the universe of all births in Gilgel Gibe in the two years prior to the estimated start date of the survey ( 2000 births). We sampled 550 households at baseline. From these 79 households were not included because the children could not be located, had died, or had moved from the study area. An additional 20 households were excluded because the father was not living in the household or could not be located. Thus, the overall response rate was $82 \%$.

A structured questionnaire was developed and administered to participants by 9 trained interviewers. Questionnaires and consent documents were developed in English then translated and back translated by native speakers into the two dominant languages in the study area: Amharic and Affan Oromo. Households were visited and all of the participants were interviewed in their house in a private area. Husbands and wives were separately interviewed using questionnaires developed specifically for men and women (refer to additional files 1 and 2 for survey questionnaires used among women in English and Amharic, respectively, and additional files 3 and 4 for questionnaires used among men in English and Amharic, respectively). Written informed consent was obtained from all participants. The Institutional Review Boards of the University of Michigan and Jimma University reviewed and approved the study protocol.

\section{Survey Domains}

Household-level FI was measured using a seven-item scale based on those used and validated previously in diverse settings in developing countries [25-27]. Women and men were separately asked whether because food ran out or they did not have enough money to buy food 
in the last three months, they: (1) worried about running out of food, (2) ran out of food, (3) reduced variety of food for children, (4) children did not have enough to eat, (5) if the respondent or other adult did not eat enough, (6) the respondent spent the whole day without food, and/or (7) the household ever had to ask others for food or money to buy food. We were most interested in household food security. We therefore analyzed internal consistency (Cronbach's Alpha 0.93), and responses of the husband and wife were summed. Maternal and paternal summed scores were also highly correlated ( $\mathrm{p}<0.01$ ) so the average was taken to represent the household's food insecurity situation. This score was divided into three food insecurity categories for the purposes of analysis: secure (score of 0-2), moderate food insecurity (score of 3-5), and severe food insecurity (score of 6-7).

Household socioeconomic status (SES) was measured through an asset index, as is standard in low-income countries [28]. The asset index was also used to measure SES in the Ethiopia Demographic and Health Survey in 2005 [29]. A set of questions about possession of material assets was asked of each household, including whether the household possessed household electricity, a television, a radio, a phone/mobile, and/or a tapedeck/ VHS/DVD player. The number of items in household possession was summed and household socioeconomic status was categorized based on whether the household was above or below the median asset ownership, which was one of the above assets per household in this sample. Women provided corresponding responses to some of the material items and these were highly associated with their partners' responses. We therefore used men's responses as estimates of the household SES.

Additionally, we controlled for the effects of age (in years; age was subdivided into three categorical variables). Information on educational status was not collected since it is known to be universally very low in the study population [17].

\section{Dependent Variables}

The neurologic disorders screening tool was derived from the World Health Organization research protocol for measuring the prevalence of neurologic disorders in developing countries [30]. A variation of this instrument was used successfully in a suburb of Addis Abba, the capital city of Ethiopia [31]. The survey instrument included questions about neurologic symptoms including: back pain, neck pain, hand/foot numbness, arm/leg weakness, seizures, diplopia, temporary blindness, difficulty speaking, movement abnormalities while still, movement abnormalities while intending movement, and carpal tunnel syndrome. Respondents were asked if they had experienced any symptom in the past week.
Answers were coded dichotomously with a 1 indicating a "yes" answer, and a zero indicating a "no" answer. Similar symptoms were aggregated for the purpose of this analysis. The neurologic indicators of interest were: seizures (respondents reporting having had a seizure in the past week), extremity weakness (respondents reporting having had either arm/hand or leg/foot weakness in the past week), extremity numbness (respondents reporting having had either arm/hand numbness or leg/ foot numbness in the past week), movement abnormalities (respondents reporting movement abnormalities either while still or while intending movement), difficulty speaking (respondents reporting having experienced either temporary or permanent difficulty speaking in the past week), carpal tunnel (respondents reporting hand pain that awoke them from sleep in the past week), vision dysfunction (respondents reporting having had diplopia or temporary vision loss in the past week), and spinal pain (respondents reporting having had back or neck pain in the past week). We aggregated our indicators to create an indicator of ND comorbidity (respondents reporting two or more NDs in the past week).

\section{Statistical Methods}

Univariate statistics were used to describe the sample and bivariate chi-square tests were used to assess relations between each of the independent variables and outcomes of interest separately by gender. We used bivariate logistic regression to assess unadjusted associations between FI and neurologic symptoms by gender, and used multivariate logistic regression to adjust for age, and household assets. The criterion for statistical significance was set at $\alpha=0.05$. All statistical tests were carried out using SAS 9.2.

\section{Results}

Complete data were available for 900 individuals (i.e. 450 men, and 450 women) for analysis. The majority of the sample $(61.8 \%)$ was between the ages of 20 and 30 . According to the median cut-off of the asset index, $47.3 \%$ of the sample was classified as having assets below the median number reported. Severe food insecurity was prevalent among $24.7 \%$ of the sample.

Overall, vision dysfunction was the most prevalent aggregate of symptoms with $60.9 \%$ of the sample reporting diplopia or temporary vision loss in the last week. Seizure was the least prevalent ND with only $6.3 \%$ of the sample reporting a seizure in the past week. $48.1 \%$ of the sample reported symptoms falling within two or more symptom clusters within the past week.

Table 1 shows univariate statistics and bivariate chisquare tests between FI and other covariates and neurological disorders among women in Jimma Zone. In chisquare models, FI was significantly associated with higher 
Table 1 Univariate and bivariate statistics of all neurologic symptom aggregates and covariates among 450 adult women in Jimma zone, Ethiopia

\begin{tabular}{|c|c|c|c|c|c|c|c|c|c|c|c|c|c|c|c|c|c|c|c|}
\hline & \multirow{2}{*}{$\begin{array}{c}\text { Total } \\
\%\end{array}$} & \multicolumn{2}{|c|}{ Seizures } & \multicolumn{2}{|c|}{$\begin{array}{l}\text { Extremity } \\
\text { Weakness }\end{array}$} & \multicolumn{2}{|c|}{$\begin{array}{l}\text { Extremity } \\
\text { Numbness }\end{array}$} & \multicolumn{2}{|c|}{$\begin{array}{c}\text { Movement } \\
\text { Abnormalities }\end{array}$} & \multicolumn{2}{|c|}{$\begin{array}{l}\text { Difficulty } \\
\text { Speaking }\end{array}$} & \multicolumn{2}{|c|}{$\begin{array}{l}\text { Carpal } \\
\text { Tunnel }\end{array}$} & \multicolumn{2}{|c|}{$\begin{array}{c}\text { Vision } \\
\text { Dysfunction }\end{array}$} & \multicolumn{2}{|c|}{$\begin{array}{l}\text { Spinal } \\
\text { Pain }\end{array}$} & \multicolumn{2}{|c|}{$\begin{array}{l}\text { Comorbid } \\
\text { Disorders }\end{array}$} \\
\hline & & $\%$ & $p$ & $\%$ & $p$ & $\%$ & $p$ & $\%$ & $p$ & $\%$ & $\mathrm{p}$ & $\%$ & $p$ & $\%$ & $p$ & $\%$ & $p$ & $\%$ & $p$ \\
\hline Overall & 100 & 5.3 & - & 29.1 & - & 23.8 & - & 18.0 & - & 5.3 & - & 12.9 & - & 42.7 & - & 11.6 & - & 42.0 & - \\
\hline Food Insecurity & & & 0.001 & & 0.282 & & 0.038 & & $<0.001$ & & 0.092 & & $<0.001$ & & $<0.001$ & & 0.123 & & $<0.001$ \\
\hline Secure & 53.8 & 3.3 & & 28.9 & & 20.3 & & 9.9 & & 4.1 & & 8.7 & & 36.4 & & 9.9 & & 36.0 & \\
\hline Moderate & 22.9 & 2.9 & & 24.3 & & 33.0 & & 23.3 & & 3.9 & & 9.7 & & 40.8 & & 9.7 & & 38.8 & \\
\hline Severe & 23.3 & 12.4 & & 34.3 & & 22.9 & & 31.4 & & 9.5 & & 25.7 & & 59.1 & & 17.1 & & 59.1 & \\
\hline Age & & & 0.502 & & 0.053 & & 0.005 & & 0.786 & & 0.351 & & $<0.001$ & & 0.004 & & 0.091 & & 0.007 \\
\hline$<30$ & 8.4 & 9.7 & & 19.4 & & 25.8 & & 16.1 & & 0.0 & & 3.2 & & 22.6 & & 9.7 & & 32.3 & \\
\hline $30-40$ & 71.6 & 5.3 & & 30.6 & & 20.8 & & 17.0 & & 6.04 & & 11.3 & & 41.9 & & 9.8 & & 40.8 & \\
\hline$>40$ & 20.0 & 4.1 & & 26.3 & & 39.2 & & 20.3 & & 6.8 & & 27.0 & & 56.8 & & 18.9 & & 59.5 & \\
\hline Asset Index* & & & 0.491 & & 0.677 & & 0.419 & & 0.164 & & 0.267 & & 0.118 & & 0.551 & & 0.059 & & 0.627 \\
\hline high & 52.7 & 4.6 & & 30.0 & & 25.3 & & 15.6 & & 4.2 & & 10.6 & & 41.4 & & 8.89 & & 40.9 & \\
\hline low & 47.3 & 6.1 & & 28.2 & & 22.1 & & 20.7 & & 6.6 & & 15.5 & & 44.1 & & 14.6 & & 43.2 & \\
\hline
\end{tabular}

* A set of material assets was asked of each household, including the possession of household electricity, a television, a radio, a phone/mobile, and/or tapedeck/ VHS/DVD player; items were summed and household's socioeconomic status was categorized based on whether the household was above or below the median asset ownership, which was one of the above assets per household.

prevalence of each outcome of interest $(p<0.050)$ except extremity weakness $(\mathrm{p}=0.282)$, difficulty speaking $(\mathrm{p}=$ $0.092)$, and spinal pain $(0.123)$. Table 2 shows the same information among men in Jimma Zone. In chi-square models, FI was significantly associated with higher prevalence of all outcomes of interest ( $p<0.050)$ except extremity weakness $(\mathrm{p}=0.256)$.

Tables 3 shows bivariate and multivariate regression models of each outcome of interest by FI and other covariates among men and women in our sample. Among women, severe FI was associated with higher odds of seizures, movement abnormalities, carpal tunnel, vision dysfunction, and spinal pain in both unadjusted and adjusted models. Severe FI was also associated with higher odds of having comorbid disorders in both unadjusted and adjusted models. Moderate FI was only associated with extremity numbness and movement abnormalities in both unadjusted and adjusted models.

Table 4 shows bivariate and multivariate regression models of each outcome of interest by FI and other covariates among men in our sample. Among men, severe FI was associated with higher odds of seizures, extremity numbness, movement abnormalities, difficulty speaking, carpal tunnel, and vision dysfunction in both unadjusted and adjusted models. Severe FI was also associated with higher odds of having comorbid disorders in both unadjusted and adjusted models. Moderate FI was only associated with extremity numbness and movement abnormalities in both unadjusted and adjusted models.

Table 5 shows comorbidity between outcomes of interest among the full sample. Generally, there was high comorbidity between outcomes. In particular, all outcomes were highly comorbid with vision dysfunction. Alternatively, outcomes were not often comorbid with seizures.

\section{Discussion}

Using data from a population-based representative sample of households in a rural area in Ethiopia, we found that FI was associated with a range of neurologic symptoms including self-reported seizure, extremity numbness, movement abnormalities, speaking difficulties, carpal tunnel syndrome, spinal pain, and vision dysfunction. FI was also associated with a higher prevalence of ND symptom comorbidity. We found that FI was generally more predictive of ND symptoms among men than among women. Finally, ND symptoms in this population were highly comorbid.

We know of no other studies that have assessed the relation between FI and risk for neurologic symptoms. However, our findings are consistent with literature documenting associations between FI and adverse health outcomes in both wealthy and less wealthy countries. For example, in a cross-sectional analysis of 724 randomly chosen low-income single women participating in welfare in the state of Michigan, USA, Siefert and colleagues [11] found that food insufficiency was associated with poor or fair self-rated health and DSM-III criteria major depressive symptoms. In a sample of 1,006 men and women in the rural US, Pheley and colleagues [12] found that FI was associated with poorer health measures before and after adjustment for demographic and healthcare access variables. Using data from the National Population Health Survey in Canada, food 
Table 2 Univariate and bivariate statistics of all neurologic symptom aggregates and covariates among 450 adult men in Jimma zone, Ethiopia

\begin{tabular}{|c|c|c|c|c|c|c|c|c|c|c|c|c|c|c|c|c|c|c|c|}
\hline & \multirow{2}{*}{$\begin{array}{c}\text { Total } \\
\%\end{array}$} & \multicolumn{2}{|c|}{ Seizures } & \multicolumn{2}{|c|}{$\begin{array}{l}\text { Extremity } \\
\text { Weakness }\end{array}$} & \multicolumn{2}{|c|}{$\begin{array}{l}\text { Extremity } \\
\text { Numbness }\end{array}$} & \multicolumn{2}{|c|}{$\begin{array}{c}\text { Movement } \\
\text { Abnormalities }\end{array}$} & \multicolumn{2}{|c|}{$\begin{array}{l}\text { Difficulty } \\
\text { Speaking }\end{array}$} & \multicolumn{2}{|c|}{$\begin{array}{l}\text { Carpal } \\
\text { Tunnel }\end{array}$} & \multicolumn{2}{|c|}{$\begin{array}{c}\text { Vision } \\
\text { Dysfunction }\end{array}$} & \multicolumn{2}{|c|}{$\begin{array}{l}\text { Spinal } \\
\text { Pain }\end{array}$} & \multicolumn{2}{|c|}{$\begin{array}{l}\text { Comorbid } \\
\text { Disorders }\end{array}$} \\
\hline & & $\%$ & $p$ & $\%$ & $p$ & $\%$ & $p$ & $\%$ & $p$ & $\%$ & $p$ & $\%$ & $p$ & $\%$ & $p$ & $\%$ & $p$ & $\%$ & $p$ \\
\hline Overall & 100 & 5.1 & - & 28.0 & - & 22.9 & - & 17.2 & - & 5.1 & - & 12.5 & - & 40.9 & - & 11.2 & - & 40.3 & - \\
\hline Food Insecurity & & & 0.003 & & 0.256 & & 0.009 & & $<0.001$ & & 0.015 & & $<0.001$ & & $<0.001$ & & 0.003 & & $<0.001$ \\
\hline Secure & 53.5 & 2.5 & & 28.1 & & 18.2 & & 9.9 & & 3.3 & & 7.4 & & 34.7 & & 9.9 & & 32.6 & \\
\hline Moderate & 18.1 & 4.9 & & 24.4 & & 31.7 & & 19.5 & & 3.7 & & 7.3 & & 35.2 & & 4.9 & & 39.0 & \\
\hline Severe & 28.3 & 10.9 & & 34.4 & & 29.7 & & 32 & & 10.2 & & 27.3 & & 63.3 & & 19.5 & & 61.7 & \\
\hline Age & & & 0.183 & & $<0.001$ & & $<0.001$ & & 0.019 & & 0.367 & & $<0.001$ & & $<0.001$ & & 0.134 & & $<0001$ \\
\hline$<20$ & 39.4 & 2.6 & & 15.8 & & 15.1 & & 11.2 & & 4.0 & & 4.6 & & 23.0 & & 8.6 & & 23.7 & \\
\hline $20-30$ & 44.8 & 4.1 & & 34.1 & & 27.8 & & 21.4 & & 6.9 & & 15.0 & & 53.2 & & 13.3 & & 50.3 & \\
\hline $31-40$ & 15.8 & 8.2 & & 41.0 & & 39.4 & & 24.6 & & 3.3 & & 23.0 & & 42.6 & & 18.0 & & 54.1 & \\
\hline$>40$ & & & & & & & & & & & & & & & & & & & \\
\hline Asset Index* & & & 0.373 & & 0.812 & & 0.832 & & 0.074 & & 0.19 & & 0.043 & & 0.159 & & 0.012 & & 0.196 \\
\hline high & 54.7 & 4.3 & & 27.5 & & 23.3 & & 14.3 & & 3.9 & & 9.7 & & 38.0 & & 8.1 & & 37.6 & \\
\hline low & 45.3 & 6.1 & & 28.5 & & 22.4 & & 20.6 & & 6.5 & & 15.9 & & 44.4 & & 15.0 & & 43.5 & \\
\hline
\end{tabular}

* A set of material assets was asked of each household, including the possession of household electricity, a television, a radio, a phone/mobile, and/or tapedeck/NHS/DVD player; items were summed and household's socioeconomic status was categorized based on whether the household was above or below the median asset ownership, which was one of the above assets per household. 
Table 3 Odds ratios and $95 \%$ confidence intervals from unadjusted and adjusted multivariable logistic regression models of neurologic symptom aggregates by food insecurity among 450 women in Jimma zone, Ethiopia

\begin{tabular}{|c|c|c|c|c|c|c|c|c|c|c|c|c|c|c|c|c|c|c|}
\hline \multirow[b]{2}{*}{ Food Insecurity } & \multicolumn{2}{|c|}{ Seizures } & \multicolumn{2}{|c|}{ Extremity Weakness } & \multicolumn{2}{|c|}{$\begin{array}{l}\text { Extremity } \\
\text { Numbness }\end{array}$} & \multicolumn{2}{|c|}{$\begin{array}{c}\text { Movement } \\
\text { Abnormalities }\end{array}$} & \multicolumn{2}{|c|}{ Difficulty Speaking } & \multicolumn{2}{|c|}{ Carpal Tunnel } & \multicolumn{2}{|c|}{ Vision Dysfunction } & \multicolumn{2}{|c|}{ Spinal Pain } & \multicolumn{2}{|c|}{ Comorbid Disorders } \\
\hline & & & & & & & & & & & & & & & & & & \\
\hline Secure & Ref & Ref & Ref & Ref & Ref & Ref & Ref & Ref & Ref & Ref & Ref & Ref & Ref & Ref & Ref & Ref & Ref & Ref \\
\hline Moderate & $\begin{array}{c}1.13 \\
(0.51-2.50)\end{array}$ & $\begin{array}{c}0.98 \\
(0.44-2.21)\end{array}$ & $\begin{array}{c}0.79 \\
(0.46-1.34)\end{array}$ & $\begin{array}{c}0.76 \\
(0.44-1.31)\end{array}$ & $\begin{array}{c}1.94 \\
(1.16-3.25)\end{array}$ & $\begin{array}{c}2.00 \\
(1.17-3.41)\end{array}$ & $\begin{array}{c}2.76 \\
(1.48-5.14)\end{array}$ & $\begin{array}{c}2.66 \\
(1.42-5.00)\end{array}$ & $\begin{array}{c}0.94 \\
(0.29-3.06)\end{array}$ & $\begin{array}{c}0.86 \\
(0.26-2.83)\end{array}$ & $\begin{array}{c}1.07 \\
(0.42-2.79)\end{array}$ & $\begin{array}{c}1.01 \\
(0.38-2.66)\end{array}$ & $\begin{array}{c}1.1 \\
(0.65-1.86)\end{array}$ & $\begin{array}{c}1.08 \\
(0.63-1.86)\end{array}$ & $\begin{array}{c}0.51 \\
(0.17-1.51)\end{array}$ & $\begin{array}{c}0.47 \\
(0.16-1.41)\end{array}$ & $\begin{array}{c}1.48 \\
(0.89-2.48)\end{array}$ & $\begin{array}{c}1.46 \\
(0.86-2.47)\end{array}$ \\
\hline Severe & $\begin{array}{c}3.64 \\
(1.95-6.81)\end{array}$ & $\begin{array}{c}3.31 \\
(1.74-6.39)\end{array}$ & $\begin{array}{c}1.28 \\
(0.79-2.09)\end{array}$ & $\begin{array}{c}1.23 \\
(0.74-2.03)\end{array}$ & $\begin{array}{c}1.17 \\
(0.67-2.03)\end{array}$ & $\begin{array}{c}1.13 \\
(0.64-1.99)\end{array}$ & $\begin{array}{c}4.16 \\
(2.31-7.51)\end{array}$ & $\begin{array}{c}4.07 \\
(2.25-7.38)\end{array}$ & $\begin{array}{c}2.44 \\
(0.99-6.06)\end{array}$ & $\begin{array}{c}2.28 \\
(0.91-5.73)\end{array}$ & $\begin{array}{c}5.10 \\
(2.75-9.42)\end{array}$ & $\begin{array}{c}4.23 \\
(2.24-7.98)\end{array}$ & $\begin{array}{c}3.65 \\
(2.34-5.69)\end{array}$ & $\begin{array}{c}3.16 \\
(1.99-5.01)\end{array}$ & $\begin{array}{c}2.41 \\
(1.31-4.41)\end{array}$ & $\begin{array}{c}2.09 \\
(1.12-3.92)\end{array}$ & $\begin{array}{c}3.73 \\
(2.40-5.82)\end{array}$ & $\begin{array}{c}3.27 \\
(2.07-5.18)\end{array}$ \\
\hline \multicolumn{19}{|c|}{ 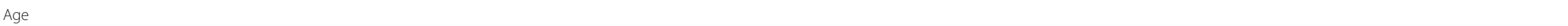 } \\
\hline$<30$ & & Ref & & Ref & & Ref & & Ref & & Ref & & Ref & & Ref & & Ref & & Ref \\
\hline $30-40$ & & $\begin{array}{c}1.70 \\
(0.74-3.89)\end{array}$ & & $\begin{array}{c}2.13 \\
(1.22-3.73)\end{array}$ & & $\begin{array}{c}0.99 \\
(0.57-1.71)\end{array}$ & & $\begin{array}{c}0.88 \\
(0.48-1.58)\end{array}$ & & $\begin{array}{c}2.37 \\
(0.67-8.37)\end{array}$ & & $\begin{array}{c}0.36 \\
(0.15-0.85)\end{array}$ & & $\begin{array}{c}0.33 \\
(0.21-0.52)\end{array}$ & & $\begin{array}{c}0.90 \\
(0.44-1.82)\end{array}$ & & $\begin{array}{c}0.41 \\
(0.26-0.65)\end{array}$ \\
\hline$>40$ & & $\begin{array}{c}4.67 \\
(1.89-11.56)\end{array}$ & & $\begin{array}{c}3.48 \\
(1.76-6.87)\end{array}$ & & $\begin{array}{c}2.41 \\
(1.24-4.67)\end{array}$ & & $\begin{array}{c}0.95 \\
(0.44-2.05)\end{array}$ & & $\begin{array}{c}2.46 \\
(0.56-10.73)\end{array}$ & & $\begin{array}{c}1.75 \\
(0.85-3.62)\end{array}$ & & $\begin{array}{c}0.69 \\
(0.38-1.23)\end{array}$ & & $\begin{array}{c}1.69 \\
(0.78-3.69)\end{array}$ & & $\begin{array}{c}1.34 \\
(0.75-2.40)\end{array}$ \\
\hline \multicolumn{19}{|l|}{ Asset Index* } \\
\hline high & & Ref & & Ref & & Ref & & Ref & & Ref & & Ref & & Ref & & Ref & & Ref \\
\hline low & & $\begin{array}{c}1.51 \\
(0.84-2.71)\end{array}$ & & $\begin{array}{c}0.95 \\
(0.62-1.44)\end{array}$ & & $\begin{array}{c}0.75 \\
(0.48-1.18)\end{array}$ & & $\begin{array}{c}1.19 \\
(0.72-1.97)\end{array}$ & & $\begin{array}{c}1.59 \\
(0.68-3.71)\end{array}$ & & $\begin{array}{c}1.35 \\
(0.75-2.42)\end{array}$ & & $\begin{array}{c}1.1 \\
(0.74-1.64)\end{array}$ & & $\begin{array}{c}1.73 \\
(0.95-3.15)\end{array}$ & & $\begin{array}{c}1.03 \\
(0.69-1.54)\end{array}$ \\
\hline
\end{tabular}

* A set of material assets was asked of each household, including the possession of household electricity, a television, a radio, a phone/mobile, and/or tapedeck/VHS/DVD player; items were summed and household's socioeconomic status was categorized based on whether the household was above or below the median asset ownership, which was one of the above assets per household. 
Table 4 Odds ratios and $95 \%$ confidence intervals from unadjusted and adjusted multivariable logistic regression models of neurologic symptom aggregates by food insecurity among $\mathbf{4 5 0}$ men in Jimma zone, Ethiopia

\begin{tabular}{|c|c|c|c|c|c|c|c|c|c|c|c|c|c|c|c|c|c|c|}
\hline \multirow[b]{2}{*}{ Food insecurity } & \multicolumn{2}{|c|}{ Seizures } & \multicolumn{2}{|c|}{ Extremity Weakness } & \multicolumn{2}{|c|}{$\begin{array}{l}\text { Extremity } \\
\text { Numbness }\end{array}$} & \multicolumn{2}{|c|}{$\begin{array}{c}\text { Movement } \\
\text { Abnormalities }\end{array}$} & \multicolumn{2}{|c|}{ Difficulty Speaking } & \multicolumn{2}{|c|}{ Carpal Tunnel } & \multicolumn{2}{|c|}{ Vision Dysfunction } & \multicolumn{2}{|c|}{ Spinal Pain } & \multicolumn{2}{|c|}{ Comorbid Disorders } \\
\hline & & & & & & & & & & & & & & & & & & \\
\hline Secure & Ref & Ref & Ref & Ref & Ref & Ref & Ref & Ref & Ref & Ref & Ref & Ref & Ref & Ref & Ref & Ref & Ref & Ref \\
\hline Moderate & $\begin{array}{c}2.19 \\
(0.60-7.95)\end{array}$ & $\begin{array}{c}2.12 \\
(0.58-7.73)\end{array}$ & $\begin{array}{c}0.92 \\
(0.52-1.64)\end{array}$ & $\begin{array}{c}0.89 \\
(0.49-1.60)\end{array}$ & $\begin{array}{c}2.30 \\
(1.31-4.06)\end{array}$ & $\begin{array}{c}2.30 \\
(1.29-4.11)\end{array}$ & $\begin{array}{c}2.40 \\
(1.21-4.79)\end{array}$ & $\begin{array}{c}2.33 \\
(1.17-4.66)\end{array}$ & $\begin{array}{c}1.21 \\
(0.31-4.65)\end{array}$ & $\begin{array}{c}1.17 \\
(0.30-4.54)\end{array}$ & $\begin{array}{c}1.13 \\
(0.51-2.50)\end{array}$ & $\begin{array}{c}0.98 \\
(0.44-2.21)\end{array}$ & $\begin{array}{c}1.21 \\
(0.75-1.93)\end{array}$ & $\begin{array}{c}1.16 \\
(0.72-1.88)\end{array}$ & $\begin{array}{c}1.94 \\
(1.16-3.25)\end{array}$ & $\begin{array}{c}0.84 \\
(0.38-1.86)\end{array}$ & $\begin{array}{c}1.13 \\
(0.70-1.82)\end{array}$ & $\begin{array}{c}1.08 \\
(0.66-1.76)\end{array}$ \\
\hline Severe & $\begin{array}{c}5.24 \\
(1.96-13.98)\end{array}$ & $\begin{array}{c}4.64 \\
(1.70-12.67)\end{array}$ & $\begin{array}{c}1.49 \\
(0.95-2.36)\end{array}$ & $\begin{array}{c}1.29 \\
(0.80-2.07)\end{array}$ & $\begin{array}{c}2.09 \\
(1.27-3.45)\end{array}$ & $\begin{array}{c}1.97 \\
(1.17-3.31)\end{array}$ & $\begin{array}{c}4.67 \\
(2.67-8.19)\end{array}$ & $\begin{array}{c}4.18 \\
(2.36-7.43)\end{array}$ & $\begin{array}{c}3.59 \\
(1.45-8.90)\end{array}$ & $\begin{array}{c}3.29 \\
(1.29-8.38)\end{array}$ & $\begin{array}{c}3.64 \\
(1.95-6.81)\end{array}$ & $\begin{array}{c}3.31 \\
(1.74-6.29)\end{array}$ & $\begin{array}{c}2.52 \\
(1.58-4.03)\end{array}$ & $\begin{array}{c}2.42 \\
(1.51-3.90)\end{array}$ & $\begin{array}{c}1.17 \\
(0.67-2.03)\end{array}$ & $\begin{array}{c}1.67 \\
(0.85-3.27)\end{array}$ & $\begin{array}{c}2.57 \\
(1.61-4.11)\end{array}$ & $\begin{array}{c}2.46 \\
(1.53-3.97)\end{array}$ \\
\hline \multicolumn{19}{|c|}{ 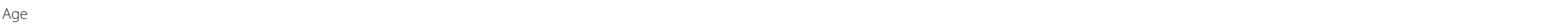 } \\
\hline$<30$ & & Ref & & Ref & & Ref & & Ref & & Ref & & Ref & & Ref & & Ref & & Ref \\
\hline $30-40$ & & $\begin{array}{c}0.57 \\
(0.18-1.78)\end{array}$ & & $\begin{array}{c}0.42 \\
(0.25-0.69)\end{array}$ & & $\begin{array}{c}0.62 \\
(0.36-1.06)\end{array}$ & & $\begin{array}{c}0.67 \\
(0.36-1.24)\end{array}$ & & $\begin{array}{c}0.79 \\
(0.30-2.13)\end{array}$ & & $\begin{array}{c}1.70 \\
(0.74-3.89)\end{array}$ & & $\begin{array}{c}1.34 \\
(0.84-2.14)\end{array}$ & & $\begin{array}{c}0.92 \\
(0.44-1.90)\end{array}$ & & $\begin{array}{c}1.39 \\
(0.86-2.22)\end{array}$ \\
\hline$>40$ & & $\begin{array}{c}1.42 \\
(0.48-5.15)\end{array}$ & & $\begin{array}{c}1.49 \\
(0.84-2.65)\end{array}$ & & $\begin{array}{c}2.13 \\
(1.17-3.88)\end{array}$ & & $\begin{array}{c}1.36 \\
(0.68-2.70)\end{array}$ & & $\begin{array}{c}0.48 \\
(0.11-2.18)\end{array}$ & & $\begin{array}{c}4.67 \\
(1.89-11.56)\end{array}$ & & $\begin{array}{c}2.31 \\
(1.25-4.27)\end{array}$ & & $\begin{array}{c}1.88 \\
(0.81-4.39)\end{array}$ & & $\begin{array}{c}2.83 \\
(1.52-5.26)\end{array}$ \\
\hline \multicolumn{19}{|l|}{ Asset Index* } \\
\hline high & & Ref & & Ref & & Ref & & Ref & & Ref & & Ref & & Ref & & Ref & & Ref \\
\hline low & & $\begin{array}{c}1.11 \\
(0.47-2.60)\end{array}$ & & $\begin{array}{c}0.95 \\
(0.63-1.45)\end{array}$ & & $\begin{array}{c}0.80 \\
(0.51-1.26)\end{array}$ & & $\begin{array}{c}1.23 \\
(0.74-2.04)\end{array}$ & & $\begin{array}{c}1.49 \\
(0.63-3.50)\end{array}$ & & $\begin{array}{c}1.51 \\
(0.84-2.71)\end{array}$ & & $\begin{array}{c}1.07 \\
(0.72-1.57)\end{array}$ & & $\begin{array}{c}1.73 \\
(0.95-3.16)\end{array}$ & & $\begin{array}{c}1.05 \\
(0.71-1.56)\end{array}$ \\
\hline
\end{tabular}

* A set of material assets was asked of each household, including the possession of household electricity, a television, a radio, a phone/mobile, and/or tapedeck/VHS/DVD player; items were summed and household's

socioeconomic status was categorized based on whether the household was above or below the median asset ownership, which was one of the above assets per household. 
Table 5 Comorbidity (in percent) between neurological disorders among 900 women and men in Jimma zone, Ethiopia*

\begin{tabular}{|c|c|c|c|c|c|c|c|c|}
\hline & Seizures & $\begin{array}{l}\text { Extremity } \\
\text { weakness }\end{array}$ & $\begin{array}{l}\text { Extremity } \\
\text { numbness }\end{array}$ & $\begin{array}{c}\text { Vision } \\
\text { dysfunction }\end{array}$ & $\begin{array}{l}\text { Difficulty } \\
\text { speaking }\end{array}$ & $\begin{array}{c}\text { Movement } \\
\text { abnormalities }\end{array}$ & $\begin{array}{c}\text { Spinal } \\
\text { pain }\end{array}$ & $\begin{array}{l}\text { Carpal } \\
\text { Tunnel }\end{array}$ \\
\hline Seizures & - & 45.8 & 41.7 & 79.2 & 12.5 & 37.5 & 37.5 & 37.5 \\
\hline Extremity weakness & 8.3 & - & 38.6 & 62.9 & 9.1 & 30.3 & 18.9 & 17.4 \\
\hline Extremity numbness & 9.3 & 47.2 & - & 52.8 & 7.4 & 39.8 & 16.7 & 27.8 \\
\hline Vision dysfunction & 9.8 & 43.0 & 29.5 & - & 9.8 & 34.7 & 18.1 & 20.7 \\
\hline Difficulty speaking & 12.5 & 50.0 & 33.3 & 79.2 & - & 54.2 & 37.5 & 45.8 \\
\hline Movement abnormalities & 11.1 & 49.4 & 53.1 & 82.7 & 16.1 & - & 24.7 & 39.5 \\
\hline Spinal pain & 17.0 & 47.2 & 34.0 & 66.0 & 17.0 & 37.7 & - & 35.9 \\
\hline Carpal Tunnel & 15.3 & 40.0 & 50.9 & 67.8 & 18.6 & 54.2 & 32.2 & - \\
\hline
\end{tabular}

*Numbers represent the percentage of those in each row with each comorbidity, by column.

insufficiency was associated with self-reported heart disease, diabetes, hypertension, food allergies, and overweight among men, as well as poorer mental and social health [13]. Among 1488 households in a rural region of the United States, FI was associated with lower selfreported physical and mental health as well as poorer health measures [32]. Another study, by Gao and colleagues, found that FI was associated with lower cognitive function in Hispanics living in the US [33]. Other studies, summarized earlier, have also shown there to be associations between FI and health in SSA [15,17].

One of the central challenges in the literature on this topic is the directionality of the association between FI and adverse health. Helping clarify this question, three longitudinal cohort studies provide evidence that is suggestive that FI or food insufficiency is a causal determinant of worse mental and physical health. Siefert and colleagues [14] found in a longitudinal analysis of 753 female public assistance recipients in the US that persistent, recurrent food insufficiency over two years was associated with higher limitations in physical functioning as compared to having only experienced FI in the present period. Heflin and colleagues [34] found in the same cohort that increases in food insufficiency predicted increases in symptoms of major depression over a three year study period. In Tanzania, Hadley and Patil [16] found that among 173 caretakers, seasonal variation in FI predicted changes in symptoms of depression and anxiety. Biological evidence also suggests that malnutrition during early neural development can cause ND in later life $[18,24]$. Conversely, however, it is also plausible that persons suffering from NDs are more likely to be disabled, and therefore unable to provide themselves with a consistent food supply. This is especially likely to be true in economies that are based heavily on subsistence agriculture. Further research will need to disentangle the causal relationship between FI and ND.

Our work also highlights the current paucity of and need for quality instruments for assessing the burden of
ND in the rural SSA setting. We found considerable discordance between the prevalences of common symptoms of ND in our sample and those reported in comparable studies. For example, we found that $60.9 \%$ (not shown) of respondents reported vision dysfunction, which compares to $1.5 \%$ and $28.6 \%$ in comparable studies $[19,35]$. Moreover, we found that $6.3 \%$ (not shown) of our sample reported seizure compared $0.7 \%$ in a comparable study [36]. Although there is no gold standard in the field, given that the prevalences of common symptoms of ND in our sample appear higher than those reported in similar studies, it is likely that our instrument overestimated the prevalences of common ND in our study population.

There are several limitations that should be considered when interpreting our findings. First, we recorded symptoms that could potentially have neurologic causes, rather than specific diagnoses of ND, and it is impossible to differentiate between neurologic, orthopedic, or other causes of many of the symptoms included in our instrument. Moreover, symptoms were not confirmed by neurologists. Therefore, the prevalences reported here should be interpreted very cautiously; it is impossible to draw conclusions about the prevalence of specific diagnoses within this population or about the specific etiologies of individual symptoms. Of particular note, for example, $60.9 \%$ of our sample reported vision dysfunction. However, because there is a high prevalence of organic eye diseases that affect vision in Ethiopia $[37,38]$, it is possible that a sizable proportion of the vision dysfunction in our population is not of neurologic etiology. Second, because we sampled only households with children, it is possible that our findings do not generalize to households without children. Third, our covariate set was limited and therefore, there may be residual confounding of the association between FI and each symptom aggregate. Of particular note, we did not collect data about dietary intake, and therefore, it is plausible that the observed associations could be confounded by malnutrition. 
It has been estimated that $33 \%$ of people living in SSA are undernourished, a widely used indicator of FI. Unfortunately, in SSA, FI is likely to increase in the coming decades $[39,40]$. Moreover, the global burden of ND is estimated to rise significantly by 2020 [18]. Therefore, studies that explore the correlates of FI and ND in SSA are needed to inform clinicians and public health practitioners about how to better serve the needs of populations in this region. Investigators interested in the social causes and effects of ND could fruitfully explore different causal mechanisms that relate FI and ND. Other avenues for future research include assessing the relation between FI and specific ICD-10 diagnosable ND and exploring the associations between FI and ND in other geographic contexts. Finally, quality instruments for measuring ND in populations in SSA are deficient, and researchers interested in the epidemiology of ND in SSA might consider creating and validating such instruments for use in future studies.

\section{Conclusions}

Among a population-based representative sample of households in a rural area in Ethiopia, FI was associated with several self-reported neurologic symptoms, including seizure, extremity numbness, movement abnormalities, speaking difficulties, carpal tunnel syndrome, spinal pain, and vision dysfunction, as well as higher prevalence of neurological symptom comorbidity. The directionality of these relations is unclear, and research about potential mechanisms relating FI and ND is needed. Finally, quality instruments for measuring ND in the SSA context are deficient, and research is needed to construct and validate such tools.

\section{Additional material}

Additional file 1: Survey questionnaire for women and children in English. The survey questionnaire used to obtain data about women in the analysis translated in English.

Additional file 2: Survey questionnaire for women and children in Amharic. The survey questionnaire used to obtain data about women in the analysis in Amharic.

Additional file 3: Survey questionnaire for men in English. The survey questionnaire used to obtain data about men in the analysis translated in English.

Additional file 4: Survey questionnaire for men in Amharic. The survey questionnaire used to obtain data about men in the analysis in Amharic.

\footnotetext{
Abbreviations

FI: Food insecurity; SSA: Sub-Saharan Africa; ND: Neurological Dysfunction GGGDS: Gilgel Gibe Growth and Development Study; SES:Socioeconomic status.
}

\section{Acknowledgements}

This project was funded by NIH grants GM07863, DA 017642, DA 022720 MH082729 and MH 078152. We thank Mr. Peter Rockers and Ms. Magdalena Paczkowski for their help and support with the data.

\section{Author details}

'Department of Epidemiology, Columbia University, New York, NY, USA. ${ }^{2}$ Columbia University College of Physicians and Surgeons, New York, NY, USA. ${ }^{3}$ Department of Public Health, University of Oxford, Oxford, UK. ${ }^{4}$ Department of Anthropology, Emory University, Atlanta, GA, USA. Jimma University, Jimma, Ethiopia. ${ }^{6}$ Department of Neurosurgery, University of Michigan Medical School, Ann Arbor, MI, USA.

\section{Authors' contributions}

AME conceived the analysis, analyzed the data, and drafted the manuscript. $\mathrm{CH}$ conceived the study, coordinated data collection, and edited the manuscript. FT, AT, and JAC coordinated data collection and edited the manuscript. SG coordinated data collection, advised on data analysis, and edited the manuscript. Finally, all authors read and approved the final manuscript

\section{Competing interests}

The authors declare that they have no competing interests.

Received: 26 July 2010 Accepted: 31 December 2010 Published: 31 December 2010

\section{References}

1. Nord M, Jemsion K, Bickel G: Prevalence of Food Insecurity and Hunger by State, 1996-1998. Food Assistance and Nutrition Research Report 1999 [http://www.ers.usda.gov/publications/FANRR2/FANRR2.PDF].

2. Bickel G, Nord M, Price C, Hamilton W, Cook J: Guide to measuring food insecurity, revised 2000. United States Department of Agriculture Report 2000 [http://www.fns.usda.gov/FSEC/FILES/FSGuide.pdf].

3. Cristofar SP, Basiotis PP: Dietary intakes and selected characteristics of women ages 19-50 years and their children ages 1-5 years by reported perception of food sufficiency. J Nutr Educ 1992, 24(2):53-58.

4. Kendall A, Olson CM, Frongillo EA Jr: Relationship of hunger and food insecurity to food availability and consumption. J Am Diet Assoc 1996, 96(10):1019.

5. Tarasuk VS, Beaton GH: Women's dietary intakes in the context of household food insecurity. J Nutr 1999, 129(3):672.

6. Kleinman RE, Murphy JM, Little M, Pagano M, Wehler CA, Regal K, Jellinek MS: Hunger in children in the United States: potential behavioral and emotional correlates. Pediatrics 1998, 101(1):E3.

7. Dubois L, Farmer A, Girard M, Porcherie M: Family food insufficiency is related to overweight among preschoolers. Soc Sci Med 2006, 63(6):1503.

8. Townsend MS, Peerson J, Love B, Achterberg C, Murphy SP: Food insecurity is positively related to overweight in women. J Nutr 2001, 131(6): 1738.

9. Adams EJ, Grummer-Strawn L, Chavez G: Food insecurity is associated with increased risk of obesity in California women. I Nutr 2003, 133(4):1070

10. Hamelin AM, Habicht JP, Beaudry M: Food insecurity: consequences for the household and broader social implications. J Nutr 1999, 129(2S Suppl):525.

11. Siefert $K$, Heflin CM, Corcoran ME, Williams DR: Food insufficiency and the physical and mental health of low-income women. Women Health 2001, 32(1-2):159.

12. Pheley AM, Holben DH, Graham AS, Simpson C: Food security and perceptions of health status: a preliminary study in rural Appalachia. J Rural Health 2002, 18(3):447.

13. Vozoris NT, Tarasuk VS: Household food insufficiency is associated with poorer health. J Nutr 2003, 133(1):120.

14. Siefert $K$, Heflin CM, Corcoran ME, Williams DR: Food insufficiency and physical and mental health in a longitudinal survey of welfare recipients. J Health Soc Behav 2004, 45(2):171.

15. Hadley C, Patil CL: Food insecurity in rural Tanzania is associated with maternal anxiety and depression. Am J Hum Biol 2006, 18(3):359. 
16. Hadley C, Patil CL: Seasonal changes in household food insecurity and symptoms of anxiety and depression. Am J Phys Anthropol 2008 , 135(2):225.

17. Hadley C, Tegegn A, Tessema F, Cowan JA, Asefa M, Galea S: Food insecurity, stressful life events and symptoms of anxiety and depression in east Africa: evidence from the Gilgel Gibe growth and development study. J Epidemiol Community Health 2008, 62(11):980-986.

18. Bergen DC, Silberberg D: Nervous system disorders: a global epidemic. Arch Neurol 2002, 59(7):1194.

19. Tamrat G, Kebede Y, Alemu S, Moore J: The prevalence and characteristics of physical and sensory disabilities in Northern Ethiopia. Disabil Rehabil 2001, 23(17):799

20. Fitaw $Y$, Boersma JM: Prevalence and impact of disability in northwestern Ethiopia. Disabil Rehabil 2006, 28(15):949.

21. Tekle-Haimanot R, Abebe M, Gebre-Mariam A, Forsgren L, Heijbel J, Holmgren G, Ekstedt J: Community-based study of neurological disorders in rural central Ethiopia. Neuroepidemiology 1990, 9(5):263.

22. Grantham-McGregor S: A review of studies of the effect of severe malnutrition on mental development. J Nutr 1995, 125(8 Suppl):2233.

23. Levitsky DA, Strupp BJ: Malnutrition and the brain: changing concepts, changing concerns. J Nutr 1995, 125(8 Suppl):2212.

24. Murray CJL, Lopez AD: The Global Burden of Disease: Global Health Statistics Boston, MA: Harvard School of Public Health; 1996.

25. Swindale A, Bilinsky P: Development of a universally applicable household food insecurity measurement tool: process, current status, and outstanding issues. J Nutr 2006, 136(5):1449.

26. Frongillo EA, Nanama S: Development and validation of an experiencebased measure of household food insecurity within and across seasons in northern Burkina Faso. J Nutr 2006, 136(5):1409.

27. Melgar-Quinonez HR, Zubieta AC, MkNelly B, Nteziyaremye A, Gerardo MF, Dunford C: Household food insecurity and food expenditure in Bolivia, Burkina Faso, And the Philippines. J Nutr 2006, 136(5):1431.

28. Filmer D, Pritchett LH: Estimating wealth effects without expenditure data-or tears: an application to educational enrollments in states of India. Demography 2001, 38(1):115.

29. Central Statistical Agency[Ethiopia], ORC Macro: Ethiopia Demographic and Health Survey 2005. 2006.

30. World Health Organization: Research protocol for measuring the prevalence of neurological disorders in developing countries. Ethiop Med J 1981, 3.

31. Haimanot RT, Abebe M, Mariam AG, Forsgren L, Holmgren G, Heijbel J, Ekstedt J: Community-based study of neurological disorders in Ethiopia: development of a screening instrument. Ethiop Med J 1990, 28(3):123.

32. Stuff JE, Casey PH, Szeto KL, Gossett JM, Robbins JM, Simpson PM, Connell C, Bogle ML: Household food insecurity is associated with adult health status. J Nutr 2004, 134(9):2330.

33. Gao X, Scott T, Falcon LM, Wilde PE, Tucker KL: Food insecurity and cognitive function in Puerto Rican adults. Am J Clin Nutr 2009 89:1197-1203.

34. Heflin CM, Siefert K, Williams DR: Food insufficiency and women's mental health: findings from a 3-year panel of welfare recipients. Soc Sci Med 2005, 61(9):1971.

35. Hladik CM, UNESCO: Tropical forests, people and food: biocultural interactions and applications to development Paris Carnforth, UK; Pearl River, N.Y.: Unesco; Parthenon Pub. Group; 1993.

36. Hjern A, Angel B, Jeppson O: Political violence, family stress and mental health of refugee children in exile. Scand J Soc Med 1998, 26(1):18-25.

37. Melese M, Alemayehu W, Bayu S, Girma T, Hailesellasie T, Khandekar R, Worku A, Courtright P: Low vision and blindness in adults in Gurage Zone, central Ethiopia. Br J Ophthalmol 2003, 87(6):677.

38. Bejiga A, Alemayehu W: Prevalence of trachoma and its determinants in Dalocha District, Central Ethiopia. Ophthalmic Epidemiol 2001, 8(2-3):119.

39. Pinstrup-Anderson P, Pandya-Lorch R: Meeting food needs in the 21st century: How many and who will be at risk? In Who will be fed in the 21st Century? Challenges for sceince and policy. Edited by: Wiebe K, Ballenger N, Pinstrup-Anderson P. Baltimore, MD: Johns Hopkins University Press; 2001:3-16.

40. Baro M, Deubel TF: Persistent Hunger: Perspectives on Vulnerability, Famine, and Food Security in Sub-Saharan Africa. Annu Rev Anthropol 2006, 35(1):521.

\section{Pre-publication history}

The pre-publication history for this paper can be accessed here: http://www.biomedcentral.com/1471-2458/10/802/prepub

doi:10.1186/1471-2458-10-802

Cite this article as: El-Sayed et al:: Household food insecurity and symptoms of neurologic disorder in Ethiopia: An observational analysis. BMC Public Health 2010 10:802.

\section{Submit your next manuscript to BioMed Central and take full advantage of:}

- Convenient online submission

- Thorough peer review

- No space constraints or color figure charges

- Immediate publication on acceptance

- Inclusion in PubMed, CAS, Scopus and Google Scholar

- Research which is freely available for redistribution

Submit your manuscript at www.biomedcentral.com/submit
Ciomed Central 